\title{
Naloxone and Metabolites Quantification in Cord Blood of Prenatally Exposed Newborns and Correlations with Maternal Concentrations
}

\author{
Samantha L. Wiegand, MD ${ }^{1}$ Madeleine J. Swortwood, PhD ${ }^{2}$ Marilyn A. Huestis, PhD ${ }^{2}$ John Thorp, MD ${ }^{1}$ \\ Hendreé E. Jones, $\mathrm{PhD}^{1}$ Neeta L. Vora, $\mathrm{MD}^{1}$
}

${ }^{1}$ Department of Obstetrics and Gynecology, University of North Carolina School of Medicine, Chapel Hill, North Carolina

2 Department of Chemistry and Drug Metabolism, Intramural Research Program, National Institute on Drug Abuse National Institutes of Health, Baltimore, Maryland

\author{
Address for correspondence Samantha L. Wiegand, MD, Berry \\ Building, One Wyoming Street, Dayton, $\mathrm{OH} 45409$, \\ (e-mail: samwiegand@gmail.com).
}

Am J Perinatol Rep 2016;6:e385-e390.

\begin{abstract}
Objective To quantify naloxone and metabolite concentrations in newborns prenatally exposed to sublingual buprenorphine/naloxone and to correlate neonatal and maternal metabolite concentrations.

Methods This is a prospective observational cohort study. Eleven pregnant women treated for opioid use disorder with sublingual buprenorphine/naloxone were enrolled. Maternal and newborn blood was collected and analyzed for naloxone, buprenorphine, and metabolites via liquid chromatography tandem mass spectrometry. Descriptive statistics and correlation coefficients were utilized to analyze data.

Results Maternal daily naloxone and buprenorphine doses were 1 to $5 \mathrm{mg}$ and 4 to $20 \mathrm{mg}$, respectively; the mean (standard deviation) time from medication until delivery was 9.9 (4.3) hours. Naloxone was below the limits of quantification (LOQ) in five infants and six mothers with a range of less than LOQ to $0.3 \mu \mathrm{g} / \mathrm{L}$. There was a strong positive correlation between maternal and newborn naloxone concentrations: Spearman's $p=0.89(p<0.01)$. There were strong positive correlations between maternal and neonatal assays for the buprenorphine analyte concentrations: buprenorphine $\rho=0.88(p<0.01)$, norbuprenorphine $\rho=0.71(p=0.01)$, and norbuprenorphine-glucuronide $\rho=0.98(p<0.01)$, but

\section{Keywords}

- buprenorphine

- naloxone

- opioid use disorder

- pregnancy

not for buprenorphine-glucuronide, $\rho=0.53(p=0.10)$.

Conclusion Naloxone and buprenorphine are transferred to the fetus during prenatal exposure to maternal sublingual buprenorphine/naloxone. The quantity of naloxone transferred from maternal circulation is minimal and highly correlated with maternal concentrations.
\end{abstract}

From 2012 to $2013,5.4 \%$ of pregnant women in the United States 15 to 44 years of age admitted to current substance use, up from $4.4 \%$ from the same survey in $2010 .^{1}$ The number of women discharged with the diagnosis of opioid use at time of delivery increased from 1.19 to 5.63 per 1,000 births annually from 2000 to 2009 , correlating with an increase in the diagnosis of neonatal abstinence syndrome (NAS) across the United States. ${ }^{2}$ Heroin use in pregnancy has been linked to fetal growth restriction, abruptio placentae, fetal death, preterm labor, and intrauterine passage of meconium; opioid use is related to an increased risk for maternal morbidities such as poor/late prenatal care, poor nutrition, sexually transmitted infections, and violence. ${ }^{3}$ received

July 9,2016

accepted after revision

September 26, 2016
DOI http://dx.doi.org/

10.1055/s-0036-1593993. ISSN 2157-6998.
Copyright $\odot 2016$ by Thieme Medical Publishers, Inc., 333 Seventh Avenue, New York, NY 10001, USA. Tel: +1(212) 584-4662.
License terms

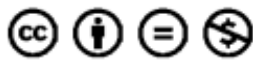


The principal treatment for opioid use disorder is medication-assisted treatment (MAT) utilizing medications such as methadone or buprenorphine along with comprehensive behavioral therapy. Treatment of opioid use disorder in pregnant women via MAT has resulted in improved adherence to prenatal care, maternal weight gain, and neonatal birth weight. ${ }^{4}$ Prenatal treatment with buprenorphine is well studied with three randomized clinical trials and 44 nonrandomized studies demonstrating its efficacy and safety compared with methadone. ${ }^{5}$ Advantages for utilizing buprenorphine compared with methadone include its office-based prescribing ability (vs. daily distribution of methadone), lower drug interaction profile, lower overdose potential, and less severe NAS. ${ }^{5}$ There is emerging information on the treatment of pregnant women utilizing the MAT combination medication buprenorphine/naloxone, ${ }^{6-9}$ which is widely used in the addiction medicine treatment community for nonpregnant individuals.

Naloxone is an opioid receptor antagonist with poor oral bioavailability that will precipitate withdrawal if insufflated or administered intravenously; therefore, it is used as an additive to sublingual buprenorphine medication formulations to deter the misuse of such medications. Naloxone is known to cross the human placenta at term after intravenous or intramuscular injection..$^{10}$ Animal models have demonstrated no teratogenic effects in the offspring of pregnant mice and hamsters given large doses of naloxone. ${ }^{11,12}$ However, some animal studies suggest alterations in fetal ovine hormone concentrations and neonatal rat behavioral effects. ${ }^{13-15}$ Therefore, clinicians have safety concerns for its use in human pregnancy.

The present study was designed to ascertain the concentrations of naloxone and metabolites measureable in the cord plasma of newborns exposed in utero to maternal sublingual buprenorphine/naloxone and to correlate these concentrations to those of the mother. Due to its poor oral absorption, our hypothesis was that no quantifiable amounts of naloxone would be detected in neonatal cord blood.

\section{Materials and Methods}

This was a prospective observational cohort study performed at University of North Carolina Hospitals (UNCH) with formal IRB approval (IRB number: 13-2699). Pregnant patients treated with buprenorphine/naloxone for opioid use disorder within the UNC Horizons Program, a gender-specific comprehensive substance use disorder treatment program, were recruited according to the following inclusion criteria: age 18 years or older, English speaking, treatment with buprenorphine/naloxone beginning at least 30 days prior to expected date of delivery, singleton fetus as confirmed by a formal ultrasound. All deliveries occurred from December 2013 to October 2014. This time period was selected to ensure all samples were less than 12 months old at the time of analysis as information regarding stability of analytes in plasma is scarce.

At the time of delivery, confirmation of buprenorphine/ naloxone dose and timing of last dose were confirmed by medication records and/or patient query. Directly after delivery, newborn cord blood and maternal blood were collected in BD Vacutainer ${ }^{\circledR}$ green-top tubes. The blood samples were centri- fuged for 15 minutes at 2,500 rpm within 30 minutes of collection. Plasma was aspirated from the samples and stored as 1-mL aliquots at $-20^{\circ} \mathrm{C}$ until analysis.

Plasma $(100 \mu \mathrm{L})$ was diluted with 2-mL $0.1 \mathrm{M}$ phosphoric acid and centrifuged prior to loading onto preconditioned strong cation-exchange polymeric solid phase extraction columns (Strata X-C, $60 \mathrm{mg} / 3 \mathrm{~mL}$ ). Analytes were eluted with 3-mL methylene chloride:isopropanol: ammonium hydroxide $(70: 26: 4, \mathrm{v} / \mathrm{v} / \mathrm{v})$, dried completely under nitrogen at $35^{\circ} \mathrm{C}$ and reconstituted in $125-\mu \mathrm{L}$ mobile phase $(85: 15, \mathrm{~A}: \mathrm{B}, \mathrm{v} / \mathrm{v})$. Extracted specimens were placed into a $4^{\circ} \mathrm{C}$ refrigerated autosampler and $50 \mu \mathrm{L}$ was injected onto a Shimadzu Prominence UFLCxr coupled to a SCIEX 5500 QTRAP mass spectrometer for analysis. Analytes were separated via gradient elution at $0.5 \mathrm{~mL} / \mathrm{min}$ with $0.1 \%$ formic acid in water $(\mathrm{A})$ and $0.1 \%$ formic acid in methanol (B) on a Restek Raptor Biphenyl column $(100 \times 2.1 \mathrm{~mm}, 2.7 \mu \mathrm{m})$ at $40^{\circ} \mathrm{C}$. Data were acquired via multiple reaction monitoring (MRM) with positive electrospray ionization (ESI); two MRMs were acquired for each analyte. The method was fully validated according to scientific working group for forensic toxicology (SWGTOX) guidelines for quantification of buprenorphine, norbuprenorphine, buprenorphine-glucuronide, norbuprenorphine-glucuronide, naloxone, nornaloxone, naloxone-glucuronide, and naloxone-N-oxide in plasma. Limits of quantification (LOQ) were 0.025 to $0.25 \mu \mathrm{g} / \mathrm{L}$ with linearity up to $50 \mu \mathrm{g} / \mathrm{L}$ (except $25 \mu \mathrm{g} / \mathrm{L}$ for naloxone and buprenorphine-glucuronide).

Maternal data collected at delivery included age, race, primary insurance, medications, gravidity, parity, smoking status, mode of delivery, and medical comorbidities. Neonatal data included gestational age at delivery, neonatal intensive care unit admission, NAS diagnosis, sex, Apgar scores at 1 and 5 minutes of life, birthweight, and medical complications. The diagnosis of NAS is standardized at UNCH; at risk infants are assessed by nursing staff utilizing a standardized 13-item opioid weaning score (total score possible 1-25). The diagnosis of NAS was made after three consecutive scores of8 or more or two consecutive scores of 12 or more.

Maternal and infant characteristics were reported utilizing descriptive statistics. Associations between maternal and newborn metabolite concentrations were examined with Spearman's correlation coefficient. Statistical significance was considered when $p<0.05$. All analyses were conducted with Stata 11.0.

\section{Results}

Eleven women were enrolled and all participated in specimen collection; cord blood was collected from all newborns. - Table 1 presents maternal and infant demographics and characteristics. All women were Caucasian, most were publicly insured, and half were smokers. The median dose of medication at delivery was 16-mg buprenorphine (range $4-20 \mathrm{mg}$ ) and 4-mg naloxone (range 1-5 mg). The mean time (standard deviation) from last dose until delivery was 9.9 (4.3) hours. Five of eleven (45\%) neonates were diagnosed with NAS.

- Table 2 presents maternal and neonatal concentrations and summary statistics for naloxone, buprenorphine, and metabolites in plasma at the time of delivery. Nornaloxone, 
Table 1 Maternal and neonatal characteristics $(N=11)$

\begin{tabular}{|l|l|}
\hline Variable & $\begin{array}{l}\text { Maternal-infant } \\
\text { dyads } \\
(N=11)\end{array}$ \\
\hline Maternal Age (years) & $28.4(5.9)$ \\
\hline Race & $11(100 \%)$ \\
\hline Caucasian & \\
\hline Insurance & $8(73 \%)$ \\
\hline Public & $3(27 \%)$ \\
\hline Private & $4[1-8]$ \\
\hline Gravidity & $2[0-6]$ \\
\hline Parity & $6(55 \%)$ \\
\hline Smoker (cigarettes) & \\
\hline Mode of delivery & $8(73 \%)$ \\
\hline Vaginal & $3(27 \%)$ \\
\hline Cesarean & $4[1-5]$ \\
\hline Naloxone dose (milligrams) & $16[4-20]$ \\
\hline Buprenorphine dose (milligrams) & $9.9(4.3)$ \\
\hline $\begin{array}{l}\text { Time from medication to } \\
\text { delivery (hours) }\end{array}$ & $1(9 \%)$ \\
\hline Preterm & $38.3(3.9)$ \\
\hline Gestational age at delivery (weeks) & $2(36 \%)$ \\
\hline Infant sex & $7(64 \%)$ \\
\hline Male & $1(9 \%)$ \\
\hline Female & $2761(817)$ \\
\hline 5-minute Apgar $<$ & $2(18 \%)$ \\
\hline Infant birthweight (grams) & $5 \%)$ \\
\hline Neonatal intensive care unit admission & \\
\hline Neonatal abstinence syndrome: yes & \\
\hline
\end{tabular}

Note: Expressed as $n$ (\%), mean (standard deviation), or median [range].

naloxone-glucuronide, and naloxone-n-oxide were also quantified but were not represented in the table as they are not biologically active. Newborn mean/median cord plasma naloxone concentrations were similar to maternal concentrations or up to $0.1 \mu \mathrm{g} / \mathrm{L}$ higher. Mean/median buprenorphine metabolite concentrations were lower in newborn cord plasma versus maternal plasma concentrations. All maternal and infant analyte concentrations demonstrated strong positive correlations with the exception of buprenorphine-glucuronide, which showed a nonstatistically significant moderate correlation $(\rho=0.53$; $p=0.10$ ). Naloxone concentrations were less than LOQ in five dyads. All buprenorphine metabolites were measurable except for norbuprenorphine concentrations in one dyad and within one newborn.

\section{Comment}

Our study demonstrates that sublingual naloxone and its metabolites are transferred transplacentally to the fetus during pregnancy and that neonatal cord blood plasma concentrations correlate with maternal plasma concentrations. Previous studies showed that naloxone is transferred when given intravenously or intramuscularly, but to the best of our knowledge, ours is the first to demonstrate transfer after sublingual naloxone administration.

Prior studies have also quantified buprenorphine in human newborn tissues and waste (umbilical cord, meconium, umbilical cord plasma) using similar analytical methods. ${ }^{16-18}$ Concheiro et $\mathrm{al}^{16}$ quantified buprenorphine and metabolites in prenatally exposed newborns and showed positive correlations with maternal concentrations, consistent with our results. However, our method demonstrates improved LOQ in a smaller sample size and shorter analysis time while simultaneously reporting on naloxone and its metabolites. The methodology used here is applicable to low-volume specimens (often the case for infant specimens), offers a wide linear range (for analysis of maternal and infant specimens with the same technique), and less costly in terms of consumables and instrument and personnel time.

Rodents exposed to high naloxone doses in utero fail to show teratogenicity ${ }^{11,12}$; human safety data regarding naloxone studies during pregnancy are nonexistent. Several observational cohort studies reviewing neonatal outcomes when their mothers were treated with buprenorphine/naloxone demonstrated outcomes similar to those whose mothers were treated with methadone or buprenorphine monotherapy. ${ }^{6-9}$ Citing lack of safety data, most authorities recommend that pregnant women who are treated with buprenorphine/ naloxone at conception switch to either buprenorphine monotherapy or methadone. ${ }^{3,19,20}$

Safety concerns may include fetal or neonatal withdrawal from the presence of naloxone in combination with opioids; adult studies show that if naloxone is given sublingually it does not affect the efficacy or pharmacological properties of buprenorphine. ${ }^{21}$ If naloxone is injected in the presence of an opioid, withdrawal is possible. Naloxone given via the sublingual route results in rapid absorption but poor bioavailability due to first-pass liver glucuronidation (to naloxone-glucuronide), $N$-dealkylation (to nornaloxone), and reduction of the 6 oxo group (to naloxone-N-oxide) to form metabolites which have not been shown to be biologically active. In contrast, all buprenorphine metabolites appear to be biologically active. ${ }^{22}$ In our study, the active medication, naloxone, was detected in the cord blood of six neonates, two of whom were diagnosed with NAS, but it was below the limit of quantification in five neonates, three of whom developed NAS. The half-life of intravenous naloxone in neonates is $3.1 \pm 0.5$ hours, ${ }^{23}$ this is in contrast to the half-life of buprenorphine in neonates, which is $\sim 11$ hours. ${ }^{24}$ If the naloxone present in the newborn circulation were enough to precipitate withdrawal, it is more likely that the neonates with detectable naloxone levels would have been diagnosed more consistently and directly after delivery. However, this was not the case.

Strengths of this study include its analytical novelty and clinical implications for further investigation. Opioid dependence and its maternal and newborn consequences are issues increasingly faced by hospitals and communities, including the resulting increase in NAS diagnosis and treatment. ${ }^{25}$ 


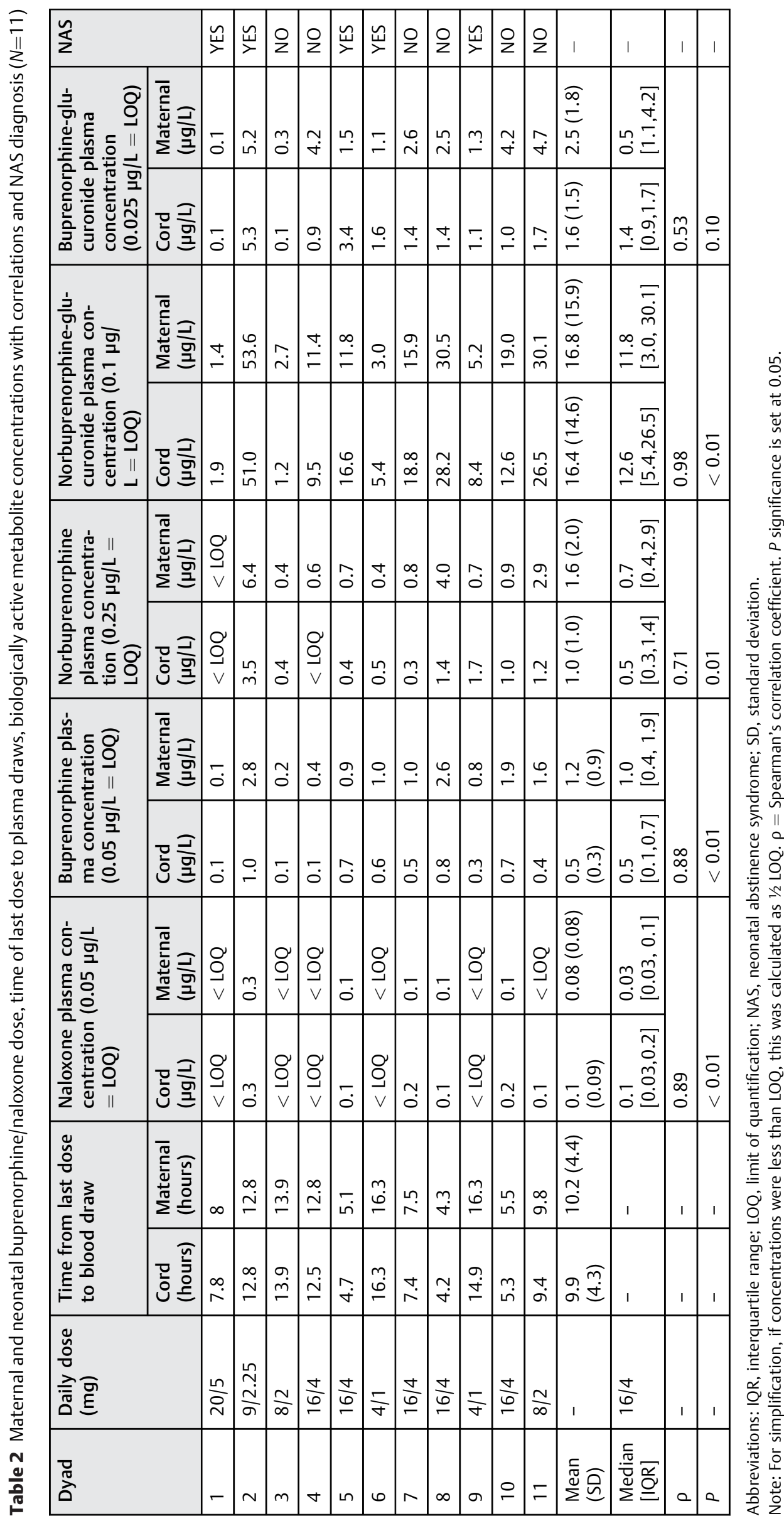


Studies that provide clinical information to women and providers can lead to more informed decision making when discussing treatment options. The U.S. Government Accountability Office published a report in February 2015, citing that one of the biggest research gaps in prenatal opioid use includes the effectiveness of various drugs and treatment of opioid use in pregnancy. ${ }^{26}$

Clinical consequences of naloxone transference in this study are unknown and the number of patients in our study is limited. The intravenous dose of naloxone for treatment of opioid overdose in both adult and pediatric populations is $0.01 \mathrm{mg} / \mathrm{kg}$-we detected neonatal levels in the range below $\mathrm{LOQ}-0.3 \mu \mathrm{g} / \mathrm{L}$, equivalent to a maximum dose of $0.0003 \mathrm{mg} /$ $\mathrm{kg}$. The results of our study may be reassuring to women who expose their fetus to naloxone prior to knowing their pregnancy status and to providers in the addiction medicine community who are looking for MAT options for pregnant patients. However, our study is limited by its small number and homogeneity (all Caucasian subjects). Data on maternal body mass index were also lacking. Future studies with larger numbers of women, maternal and neonatal clinical consequences of buprenorphine/naloxone, and long-term outcomes of children exposed to buprenorphine/naloxone would assist in informing providers and patients of the safety and acceptability of this medication during pregnancy.

\section{Note}

Supported by a grant (Dr. Wiegand) from the Cefalo-Bowes Young Researcher Award Fund, Center for Maternal and Infant Health, The University of North Carolina, Chapel Hill, North Carolina; Building Interdisciplinary Research Careers in Women's Health K12 HD001441-16 (Dr. Vora).

The content is solely the responsibility of the authors and does not necessarily represent the official views of the National Institutes of Health or Health and Human Services Administration.

The authors report no conflicts of interest.

Presented at Society of Maternal-Fetal Medicine 36th Annual Meeting, February 1-6, 2016, Atlanta, Georgia.

\section{Financial Disclosure}

The authors do not report any potential conflicts of interest.

\section{References}

1 Substance Abuse and Mental Health Services Administration. Results from the 2013 National Survey on Drug Use and Health: Summary of National Findings, NSDUH Series H-48, HHS Publication No. (SMA) 14-4863. Rockville, MD: Substance Abuse and Mental Health Services Administration; 2014

2 Patrick SW, Schumacher RE, Benneyworth BD, Krans EE, McAllister JM, Davis MM. Neonatal abstinence syndrome and associated health care expenditures: United States, 2000-2009. JAMA 2012; 307(18):1934-1940

3 ACOG Committee on Health Care for Underserved Women; American Society of Addiction Medicine. ACOG Committee Opinion No.
524: opioid abuse, dependence, and addiction in pregnancy. Obstet Gynecol 2012;119(5):1070-1076

4 Center for Substance Abuse Treatment. U.S. Department of Health and Human Services. Medication-assisted treatment for opioid addiction during pregnancy. In: SAHMSA/CSAT Treatment Improvement Protocols. Rockville, MD: Substance Abuse and Mental Health Services Administration; 2008

5 Jones HE, Heil SH, Baewert A, et al. Buprenorphine treatment of opioid-dependent pregnant women: a comprehensive review. Addiction 2012;107(Suppl 1):5-27

6 Debelak K, Morrone WR, O'Grady KE, Jones HE. Buprenorphine + naloxone in the treatment of opioid dependence during pregnancy-initial patient care and outcome data. Am J Addict 2013;22(3): 252-254

7 Lund IO, Fischer G, Welle-Strand GK, et al. A comparison of buprenorphine+naloxone to buprenorphine and methadone in the treatment of opioid dependence during pregnancy: maternal and neonatal outcomes. Subst Abuse 2013; 7:61-74

8 Wiegand SL, Stringer EM, Stuebe AM, Jones H, Seashore C, Thorp J. Buprenorphine and naloxone compared with methadone treatment in pregnancy. Obstet Gynecol 2015;125(2): 363-368

9 Gawronski KM, Prasad MR, Backes CR, Lehman KJ, Gardner DK, Cordero L. Neonatal outcomes following in utero exposure to buprenorphine/naloxone or methadone. SAGE Open Med 2014; 2:2050312114530282

10 Hibbard BM, Rosen M, Davies D. Placental transfer of naloxone. Br J Anaesth 1986;58(1):45-48

11 Geber WF, Schramm LC. Congenital malformations of the central nervous system produced by narcotic analgesics in the hamster. Am J Obstet Gynecol 1975;123(7):705-713

12 Jurand $A$. The interference of naloxone hydrochloride in the teratogenic activity of opiates. Teratology 1985;31(2): 235-240

13 Shepanek NA, Smith RF, Tyer ZE, Royall GD, Allen KS. Behavioral and neuroanatomical sequelae of prenatal naloxone administration in the rat. Neurotoxicol Teratol 1989;11(5):441-446

14 Vorhees CV. Effects of prenatal naloxone exposure on postnatal behavioral development of rats. Neurobehav Toxicol Teratol 1981; 3(3):295-301

15 Cuttler L, Egli CA, Styne DM, Kaplan SL, Grumbach MM. Hormone ontogeny in the ovine fetus. XVIII. The effect of an opioid antagonist on luteinizing hormone secretion. Endocrinology 1985; 116(5):1997-2002

16 Concheiro M, Jones HE, Johnson RE, Choo R, Shakleya DM, Huestis MA. Umbilical cord monitoring of in utero drug exposure to buprenorphine and correlation with maternal dose and neonatal outcomes. J Anal Toxicol 2010;34(8):498-505

17 Concheiro M, Jones H, Johnson RE, Shakleya DM, Huestis MA. Confirmatory analysis of buprenorphine, norbuprenorphine, and glucuronide metabolites in plasma by LCMSMS. Application to umbilical cord plasma from buprenorphine-maintained pregnant women. J Chromatogr B Analyt Technol Biomed Life Sci 2010; 878(1):13-20

18 Concheiro M, Shakleya DM, Huestis MA. Simultaneous quantification of buprenorphine, norbuprenorphine, buprenorphine-glucuronide and norbuprenorphine-glucuronide in human umbilical cord by liquid chromatography tandem mass spectrometry. Forensic Sci Int 2009;188(1-3):144-151

19 Wong S, Ordean A, Kahan M; Society of Obstetricians and Gynecologists of Canada. SOGC clinical practice guidelines: Substance use in pregnancy: no. 256, April 2011. Int J Gynaecol Obstet 2011; 114(2):190-202

20 Kampman K, Jarvis M. American Society of Addiction Medicine (ASAM) National Practice Guideline for the Use of Medications in the Treatment of Addiction Involving Opioid Use. J Addict Med 2015;9(5):358-367 
21 Chiang CN, Hawks RL. Pharmacokinetics of the combination tablet of buprenorphine and naloxone. Drug Alcohol Depend 2003;70, (2 Suppl):S39-S47

22 Brown SM, Holtzman M, Kim T, Kharasch ED. Buprenorphine metabolites, buprenorphine-3-glucuronide and norbuprenorphine-3-glucuronide, are biologically active. Anesthesiology 2011;115(6):1251-1260

23 Mooreland TA, Brice JEH, Walker CHM, Parija AC. Naloxone pharmacokinetics in the newborn. Br J Clin Pharmacol 1980; 9:609-612
$24 \mathrm{Ng} \mathrm{CM}$, Dombrowsky E, Lin H, et al. Population pharmacokinetic model of sublingual buprenorphine in neonatal abstinence syndrome. Pharmacotherapy 2015;35(7):670-680

25 Tolia VN, Patrick SW, Bennett MM, et al. Increasing incidence of the neonatal abstinence syndrome in U.S. neonatal ICUs. N Engl J Med 2015;372(22):2118-2126

26 Government Accountability Office. Prenatal Drug Use and Newborn Health. Federal Efforts Need Better Planning and Coordination. (GAO Publication No. 15-203). Washington, DC: U.S. Government Printing Office; 2015 